\title{
Nitrogen-doped coatings on carbon nanotubes and their stabilizing effect on Pt nanoparticles $\nmid$
}

\author{
Xenia Tuaev, ${ }^{a}$ Jens Peter Paraknowitsch, ${ }^{a}$ René Illgen, ${ }^{b}$ Arne Thomas ${ }^{a}$ and Peter Strasser ${ }^{a}$ \\ Received 16th January 2012, Accepted 15th March 2012 \\ DOI: $10.1039 / \mathrm{c} 2 \mathrm{cp} 40760 \mathrm{~d}$
}

\begin{abstract}
A homogeneous coating of nitrogen-doped carbon on carbon nanotubes is performed using ionic liquids. The $\mathrm{N}$-doped material is employed as a support for nanoparticles. Electrochemical degradation behavior is monitored in situ and compared to an unmodified material. The strongly enhanced stability is explained on the basis of a Pt-nitrogen interaction.
\end{abstract}

Metal nanoparticles (NPs) are a frequently employed catalytically active component of industrial catalysts such as three-way catalysts in catalytic converters, reforming catalysts in refinery processes or electrode catalysts in electrochemical devices. This is because nanoparticles offer a large catalytically active surface area, which is indispensable for cost- and material efficient product yields. In electrocatalytic electrodes, such as polymer electrolyte membrane (PEM) fuel cell electrodes or PEM electrolyzer electrodes, highly dispersed metal nanoparticles ensure sufficient electrochemical surface areas (ECSA) and hence viable electrochemical power densities and hydrogen yields, respectively. However, the dispersed nanoparticles show structural instabilities due to their high surface energy. ${ }^{1-8}$ Ex situ transmission electron microscopy (TEM) studies ${ }^{4,8-11}$ have evidenced that electrocatalytic Pt nanoparticles quickly grow and coarsen; this manifests itself as degradation of cell voltage and loss in the ECSA. The growth rate was found to be dependent on the surface area of the carbon support with high surface area carbons showing a slower Pt coarsening rate than low surface area supports. ${ }^{5,6}$ In parallel to the particle coarsening, carbon supports corrode and degrade due to the high cathodic electrode potentials. Much has been learned about the molecularscale degradation mechanisms of fuel cell cathodes, ${ }^{8}$ however, to date, robust and practical material-design strategies to mitigate the structural degradation of carbon-supported Pt nanoparticle catalysts has remained scarce.

\footnotetext{
${ }^{a}$ Technical University Berlin, Department of Chemistry,

Department of Material Science, Strasse des 17ten Juni 124,

10623 Berlin, Germany.E-mail: xenia.tuaev@tu-berlin.de,

pstrasser@tu-berlin.de; Fax: + 4930314 22261; Tel: + 493027883

${ }^{b}$ Federal Institute for Materials Research and Testing (BAM)

Division 6.4, Unter den Eichen 44-46, 12203 Berlin, Germany

$\dagger$ Electronic supplementary information (ESI) available: Experimental procedures, synthesis of N-CNTs, synthesis of Pt-NPs on N-CNTs, diffraction patterns and electrochemical protocols. See DOI: 10.1039/ c2cp40760d
}

Here, we present a simple, but very effective strategy to mitigate Pt NP coarsening in supported high surface area fuel cell electrodes. Our study is based on the hypothesis that surface heteroatoms, such as nitrogen, mediate the enhancement of platinum adsorption on the surface of carbon materials and slow down or even suppress its growth and surface area degradation. ${ }^{12-16}$ Nitrogen doping of carbon electrodes can be achieved via a variety of synthesis methods including pyrolysis and CVD of nitrogen rich precursors and post-synthetic methods like ion bombardment or thermal treatment in the presence of reactive nitrogen compounds such as ammonia. ${ }^{16}$ The method we have used throughout this study does - in contrast - not change the backbone of the material itself, but applies a thin coating of $\mathrm{N}$-doped carbon on the surface of the electrocatalyst support via a post-synthetic method. On the one hand, this allows for the use of cheap and widely available bulk carbons as host material and on the other hand offers the possibility of a more direct comparison of doped and non-doped catalyst supports. Herein we show that by our method, growth and coarsening of $\mathrm{Pt}$ nanoparticles during electrochemical potential cycling are largely suppressed. We also show that the presence of $\mathrm{N}-\mathrm{C}$ coatings does not compromise the electrocatalytic activity of $\mathrm{Pt}$ nanoparticles. The $\mathrm{N}-\mathrm{C}$ coatings were formed from ionic liquid precursors, which can wet the surface of the pristine carbon support and subsequently be pyrolyzed into nitrogen-rich carbon coatings. Particle growth on the pristine and $\mathrm{N}-\mathrm{C}$ coated carbons was monitored using time-resolved in situ Small Angle X-ray Scattering (in situ SAXS), ${ }^{17,18}$ a non-invasive powerful, yet rarely used method to monitor growth trajectories of electrocatalytic particles in real time.

Ionic liquids are established precursor compounds in the field of materials chemistry, ${ }^{19-23}$ but just recently they have been applied as precursors for nitrogen-doped carbon. ${ }^{24-29}$ As liquids with negligible vapour pressure, such precursors can be easily processed and transformed into $\mathrm{N}$-doped carbon materials by simple annealing under inert gas atmosphere. ${ }^{26,27} \mathrm{~N}$-doped carbons derived from 3-methyl- $N$-butyl-pyridinium-dicyanamide (3MBP-dca) exhibit interesting properties: even at carbonisation temperatures of $1000{ }^{\circ} \mathrm{C}$ a high nitrogen content of approx. $10 \mathrm{wt} \%$ is observed in the resulting carbon material. The nitrogen atoms are incorporated into the carbon backbone in pyridinic or quaternary graphitic bonding environments within a structure that is dominated by graphitic microdomains. Furthermore, remarkable electric conductivities were reported, reaching the range of highly ordered graphite. ${ }^{26,27}$ Thus IL-derived 
$\mathrm{N}$-doped carbons have been envisaged as interesting materials for electrochemical applications.

Unfortunately the usage of ILs as carbon precursors is accompanied with relatively high costs especially in comparison to the low price of most other carbon precursors. Thus to exploit the favourable properties of IL derived carbons in a most economical way, it was envisaged to prepare just thin coatings of the IL derived carbons on a convenient bulk carbon material. Such a coating also offers the opportunity to combine the properties of the IL derived carbon with the ones of the host. One interesting example has recently been presented using IL-derived $\mathrm{N}$-doped carbon- $-\mathrm{Li}_{4} \mathrm{Ti}_{5} \mathrm{O}_{12}$ composites as cathode material in lithium ion batteries. ${ }^{30}$

In this study the IL 3MBP-dca was used to prepare a $\mathrm{N}$-doped carbon ( $\mathrm{N}-\mathrm{C}$ ) coating on the walls of carbon nanotubes (CNTs). ${ }^{26,27}$ CNTs have been selected as a host, as their defined morphology allows for a good control of the homogeneity of the coating. Furthermore a preferable interaction of CNTs with ILs is known from the recent literature. ${ }^{31}$ It should be however noted that in principle all other forms of carbons, like activated charcoal, should be as well feasible as host materials. Prior to the application of the N-doped carbon coatings, the CNT supports were surface oxidised using sulfuric acid in order to increase the number of polar end groups. This served to even further enhance the interaction between the polar ILs with the CNT surface. IL precursor concentrations were carefully adjusted to enable a homogeneous coating and to minimize any intertubular residues (see ESI $\dagger$ for detailed experimental procedures). An optimized $30 \mathrm{~mol} \%$ of $3 \mathrm{MBP}$-dca in water was applied in the wetting of the CNTs and subsequent formation of an $\mathrm{N}$-doped carbon layer by thermal treatment under inert gas at $1000{ }^{\circ} \mathrm{C}$. Higher concentrations of ILs or even pure ILs led to strong intertubular bulk carbon contributions, while lower concentrations did not yield sufficiently and homogeneously coated materials. The resulting composite material (henceforth referred to as "N-CNT" was analysed by means of transmission electron microscopy (TEM), nitrogen sorption and X-ray photoelectron spectroscopy (XPS). Nitrogen sorption isotherms (see Fig. S1, ESI $\dagger$ ) reveal a decrease of the surface area upon hydroxylation of the CNTs, probably due to increased intertubular interactions. In N-CNTs this effect is no longer observed due to the N/C-modified CNT surfaces. The micrograph in Fig. 1a further evidences no significant amounts of excess intertubular bulk residue. A similarly heat treated non-modified CNTs material, henceforth referred to as "CNTs", were used as a benchmark support in this study.

The XPS survey scan in Fig. 1 shows peaks for C, O and N with concentrations of 97,2 and 1 at.\%, respectively. The nitrogen content may seem small at first glance. Nevertheless it has to be considered that a coating of carbon doped with $\sim 10 \mathrm{wt} \% \mathrm{~N}$ has been applied on a carbon surface; thus higher $\mathrm{N}$-contents can hardly be expected. High resolution analysis of the N1s region shows 3 components with binding energies of $398.7 \mathrm{eV}, 401.1 \mathrm{eV}$ and $404.0 \mathrm{eV}$ and with relative $\mathrm{N}$ intensities of $35.7,61.8$ and $2.5 \%$. Based on a previous study ${ }^{26}$ it was possible to identify these three components as pyridinic, graphitic and oxidised $\mathrm{N}$-species. The high resolution $\mathrm{Cls}$ region shows two loss- and the $\mathrm{sp}^{2}$ - and $\mathrm{sp}^{3}$-carbon peaks, as they are typical for CNTs. Additionally there were three
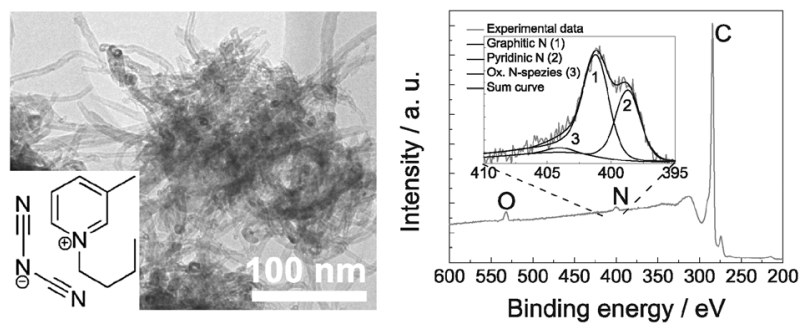

Fig. 1 Transmission electron micrographs of N-CNTs with a chemical formula of the IL-coating precursor. XP survey spectrum of coated nanotubes with a highly resolved N1s spectrum.

component peaks with additional components with binding energies of $286.8 \mathrm{eV}, 287.9 \mathrm{eV}$ and $288 \mathrm{eV}$. A specific assignment to functional groups, however, was not possible, because oxygenand nitrogen-containing groups lead to very similar chemical shifts in that region.

To study the effect of $\mathrm{N}$-doped interlayers on the electrochemically induced growth and surface area degradation of catalytic NPs, Pt nanoparticles were deposited ${ }^{32,33}$ on the benchmark CNTs as well as on the N-CNTs support materials. Particle growth trajectories were measured in situ using SAXS. SAXS offers a time resolved analysis of the particle size distribution, characterized by the mean diameter and dispersion, the number density and the volume fraction of scattering centers. ${ }^{34}$ Fig. 2a shows the high resolution TEM images of the as-prepared Pt-NP supported on the uncoated CNTs. The particles are homogeneously distributed on the support; a mean particle size of $1.7 \mathrm{~nm}$ was obtained from the log-normal distribution of the particle size resulting from counting 200 particles on TEM images (Fig. 2b).
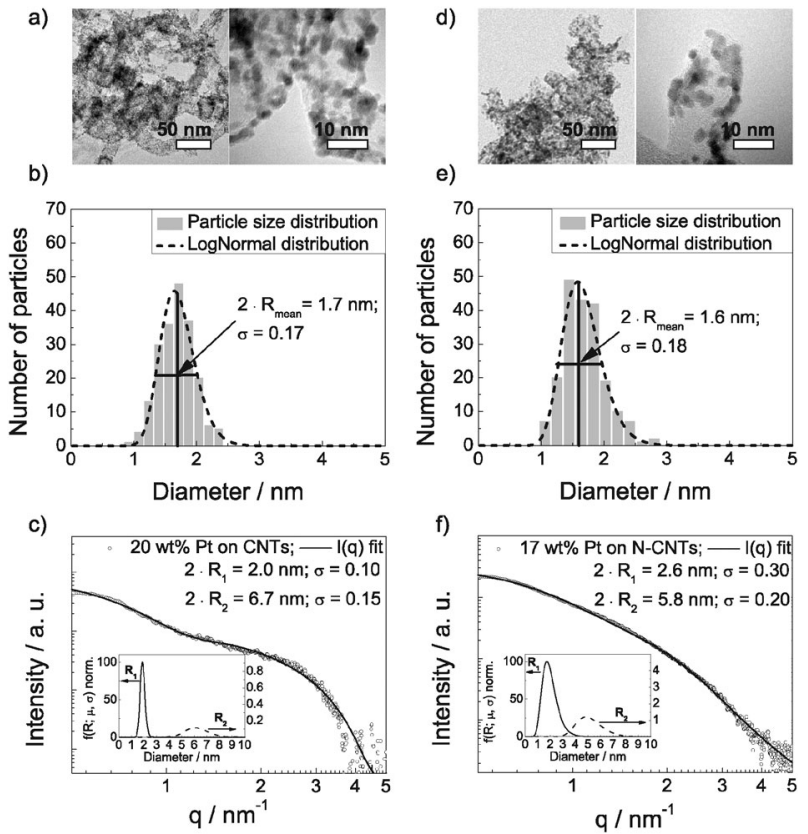

Fig. 2 High resolution TEM images of (a) $20 \mathrm{wt} \% \mathrm{Pt} /$ (unmodified) CNTs and (b) a $17 \mathrm{wt} \% \mathrm{Pt} / \mathrm{N}-\mathrm{CNT}$ catalyst. (b) and (e) show the TEM based size histograms together with a log-normal fit. Histograms were based on 200 particles. (c) and (d) show experimental SAXS profiles with a log-normal fit for the $20 \mathrm{wt} \% \mathrm{Pt} / \mathrm{CNTs}$ and the $17 \mathrm{wt} \% \mathrm{Pt} / \mathrm{N}-\mathrm{CNTs}$, respectively. Insets in (c) and (f) show the extracted bimodal particle size distributions plotted on two separate $y$ scales. 
Probing a much larger particle population than TEM, SAXS provides statistically more reliable size information. Fig. 2c shows the background-corrected scattering curve of the pristine powder and its fit using a spherical particle form factor. The inset presents the corresponding bimodal particle size distribution (psd). The psd is characterized by a narrow distribution of smaller $(\sim 2.0 \mathrm{~nm})$ and larger particles $(\sim 6.7 \mathrm{~nm})$. A similar analysis for the as-prepared Pt-NP supported on the N-CNTs is shown in Fig. 2d, e and f. The TEM derived mean particle size of the NPs was $1.9 \mathrm{~nm}$, while SAXS data again suggested a bimodal size distribution clustering around $2.6 \mathrm{~nm}$ and $5.8 \mathrm{~nm}$.

The crystallinity of the supported particles was probed by X-ray diffraction (Fig. S2, ESI $\dagger$ ). Crystallite mean size analyses $(2.1 \mathrm{~nm}$ for Pt-NPs on CNTs and $2.8 \mathrm{~nm}$ for Pt-NPs on N-CNTs) are reasonably consistent with TEM and SAXS-derived values.

Finally, the as-prepared CNT and N-CNT supported $\mathrm{Pt}-\mathrm{NPs}$ powder catalysts were used to prepare a liquid catalyst ink suspension, cast into a thin catalyst electrode film and subsequently subjected to an electrochemical voltage cycling protocol to evaluate differences in the stability of the two nanoparticle catalyst-support couples. Emphasis was placed on the evolution of the mean particle size, the ECSA and the Pt-mass based catalytic activity for the oxygen reduction reaction (ORR) to water. This reaction occurs at fuel cell cathodes, the electrode potential of which can vary between $0.5 \mathrm{~V}$ and $1.5 \mathrm{~V}$ versus the hydrogen anode depending on the fuel cell operating conditions. ${ }^{4}$ Linear sweep voltammetry (Fig. S3a and S3d, ESI $\dagger$ ) revealed Pt-mass based activities of $0.087 \mathrm{Amg}^{-1}$ for Pt-NPs supported on CNTs and $0.082 \mathrm{Amg}^{-1}$ for Pt-NPs on N-CNTs, which are in agreement with those of commercial carbon-supported Pt electrocatalysts. ${ }^{6}$ This shows that the $\mathrm{N}-\mathrm{C}$ coatings had no significantly detrimental effect on the electrocatalytic ORR activity. Cyclic voltammetry (CV) (Fig. S3b and S3e, ESI $\dagger$ ) of catalyst electrode films in a degassed electrolyte yielded time-resolved ECSA trajectories shown in Fig. 3a and d. The ECSA loss is a macroscopic descriptor for the total catalytically active surface area and is affected by a large number of different factors, such as particle dispersion, support structure, and the accessibility of particles on the support surface. With initial ECSA values in the $50-60 \mathrm{~m}^{2} \mathrm{~g}^{-1}$ range, ${ }^{5,6}$ both catalysts showed similar ECSA losses over the 2400 potential cycles of $33 \%$ for the uncoated CNT and somewhat reduced $27 \%$ for the N-CNT supported particles.

To test our hypothesis of decreased particle coarsening on $\mathrm{N}$-CNT supports, we performed in situ SAXS measurements monitoring particle size changes during voltage cycling. Background-corrected scattering $I(q)$ curves of Pt-NPs on CNTs and N-CNTs are given in Fig. S3c and S3f (ESI $\dagger$ ), respectively. Shifts of the decreasing portions of the scattering profiles towards smaller $q$ values over time, seen in Fig. S3c (ESI $\dagger$ ), evidence particle coarsening. ${ }^{17,35}$ Using the $I(q)$ data, time-resolved particle size distributions, time trajectories of correlation length particle diameters $\left(2 R_{\mathrm{lc}}\right)$ and normalized number densities of scattering centers $\left(N_{0}\right)$ were extracted and compared for the CNT and N-CNT supports (see Fig. 3b, c, e, and f, respectively). Pt-NP number density normalized diameters (see ESI $\dagger$ ) on unmodified CNTs rapidly increased from $3.8 \mathrm{~nm}$ to about $5.5 \mathrm{~nm}$ during the voltage cycling test. Simultaneously the normalized
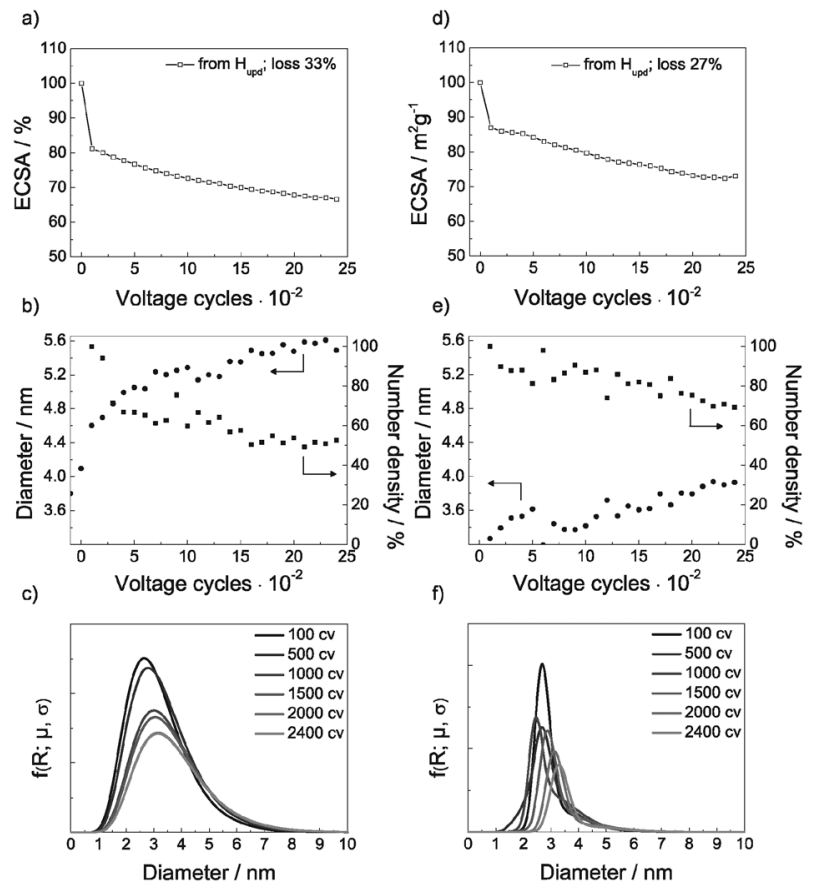

Fig. 3 (a, d) Experimental active surface area (ECSA); (b, e) in situ particle diameter change; (c, f) normalized number density of particles during potential cycling of Pt nanoparticles supported on an un-modified CNT support ( $\mathrm{a}, \mathrm{b}$ and $\mathrm{c}$ ) and on a nitrogen-carbon coated CNT support (N-CNT) (d, e and f).

number density of particles decreased dramatically by about $50 \%$, suggesting a reduction in the number of particles due to coarsening.

The time trajectories of the N-CNT supported nanoparticles offer a stark contrast to those supported on the unmodified CNT. The mean particle size increased from $3.2 \mathrm{~nm}$ to only $3.9 \mathrm{~nm}$, a mere $22 \%$ growth compared to the $45 \%$ growth of the CNT-supported particles. The size distribution function remains nearly unchanged during the potential protocol (Fig. 3f). Also, rather than a steep exponential drop, the particle number density exhibited a gradual decrease by only $30 \%$.

The observed evolution of the SAXS-derived structural parameters of the $\mathrm{Pt}$ nanoparticles strongly suggests that, owing to its IL pyrolysis coating, the N-CNT support can provide improved structural stability of a Pt particle ensemble and reduce $\mathrm{Pt}$ particle coarsening during potential cycling. Based on the measured ECSA values, a mere increase in the surface area of the N-CNT support can be excluded as the origin of the stabilization. For the given initial particle diameters, the observed ECSA losses of about $30 \%$ appear to be poorly correlated with particle growth, and rather controlled by structure of the catalyst and the support as well as the interaction with the electrolyte, respectively. Carbon corrosion, e.g., is a process which contributes to the ECSA loss, but not to the particle size observed with SAXS. The pyrolysis of ILs is known to form pyridinic, pyrrolic, and other nitrogen containing functional surface sites, often at graphene sheet edges. ${ }^{36-40}$ A stabilizing interaction between such nitrogen-containing surface regions and small Pt nanoparticles could be the explanation for reduced dissolution/Ostwald-type ripening and/or reduced migration and coalescence. Surface nitrogen thereby raises the 
dissolution potential of $\mathrm{Pt}$ making the formation of metal ions, which are migrating intermediates in the Ostwald ripening process, more difficult.

\section{Conclusions}

In summary, we have prepared a N-doped carbon nanotube material (N-CNT) by pyrolyzing an IL on the surface on a commercial CNT material. The pyrolysis products formed a thin $\mathrm{N}-\mathrm{C}$ coating on the carbon nanotubes. We have used the $\mathrm{N}-\mathrm{CNT}$ as support for Pt nanoparticles, the ORR performance of which was identical to that on unmodified CNT supports. In situ surface area measurements and particle size trajectories suggested a significantly reduced particle coarsening and degradation behavior. The surface nitrogen thus mediates the enhancement of platinum adsorption on the surface of carbon materials. Our results suggest that, more generally, $\mathrm{N}$-doped carbons offer a promising family of porous support materials for electrodes, which operate at corrosive potentials and hence are prone to dissolutionreprecipitation of a catalytically active metallic component.

\section{Acknowledgements}

This work was supported by the Cluster of Excellence in Catalysis (UNICAT) funded by the German National Science Foundation (DFG) and managed by the Technical University Berlin. Portions of this research were carried out at the SLAC National Accelerator Laboratory, a national user facility operated by Stanford University.

\section{Notes and references}

1 P. J. Ferreira, G. J. la O, Y. Shao-Horn, D. Morgan, R. Makharia, S. Kocha and H. A. Gasteiger, J. Electrochem. Soc., 2005, 152, A2256-A2271.

2 J. Xie, D. L. Wood, K. L. More, P. Atanassov and R. L. Borup, J. Electrochem. Soc., 2005, 152, A1011-A1020.

3 R. L. Borup, J. R. Davey, F. H. Garzon, D. L. Wood and M. A. Inbody, J. Power Sources, 2006, 163, 76-81.

4 R. Borup, J. Meyers, B. Pivovar, Y. S. Kim, R. Mukundan, N. Garland, D. Myers, M. Wilson, F. Garzon, D. Wood, P. Zelenay, K. More, K. Stroh, T. Zawodzinski, J. Boncella, J. E. McGrath, M. Inaba, K. Miyatake, M. Hori, K. Ota, Z. Ogumi, S. Miyata, A. Nishikata, Z. Siroma, Y. Uchimoto, K. Yasuda, K. I. Kimijima and N. Iwashita, Chem. Rev., 2007, 107, 3904-3951.

5 F. Hasché, M. Oezaslan and P. Strasser, ChemCatChem, 2011, 3, 1803-1813.

6 F. Hasché, M. Oezaslan and P. Strasser, Phys. Chem. Chem. Phys., 2010, 12, 15251-15258.

7 S. S. Zhang, X. Z. Yuan, J. N. C. Hin, H. J. Wang, K. A. Friedrich and M. Schulze, J. Power Sources, 2009, 194, 588-600.

8 Y. Shao-Horn, W. C. Sheng, S. Chen, P. J. Ferreira, E. F. Holby and D. Morgan, Top. Catal., 2007, 46, 285-305.

9 K. J. J. Mayrhofer, J. C. Meier, S. J. Ashton, G. K. H. Wiberg, F. Kraus, M. Hanzlik and M. Arenz, Electrochem. Commun., 2008, 10, 1144-1147.
10 P. J. Ferreira and Y. Shao-Horn, Electrochem. Solid-State Lett., 2007, 10, B60-B63.

11 E. F. Holby, W. C. Sheng, Y. Shao-Horn and D. Morgan, Energy Environ. Sci., 2009, 2, 865-871.

12 Y. Chen, J. Wang, H. Liu, R. Li, X. Sun, S. Ye and S. Knights, Electrochem. Commun., 2009, 11, 2071-2076.

13 Y.-H. Li, T.-H. Hung and C.-W. Chen, Carbon, 2009, 47, $850-855$.

14 C. E. Chan-Thaw, A. Villa, P. Katekomol, D. Su, A. Thomas and L. Prati, Nano Lett., 2010, 10, 537-541.

15 C. E. Chan-Thaw, A. Villa, L. Prati and A. Thomas, Chem.-Eur. J., 2011, 17, 1052-1057.

16 Y. Zhou, K. Neyerlin, T. S. Olson, S. Pylypenko, J. Bult, H. N. Dinh, T. Gennett, Z. Shao and R. O. Hayre, Energy Environ. Sci., 2010, 3, 1437-1446.

17 X. Tuaev and P. Strasser, in Polymer electrolyte membrane and direct methanol fuel cell technology, ed. C. Roth and C. Hartnig, Woodhead Publishing Series in Energy, London, 2011, vol. II: In situ characterization techniques for low temperature fuel cells.

18 C. Yu, S. Koh, J. Leisch, M. T. Toney and P. Strasser, Faraday Discuss., 2008, 140, 283-296.

19 M. Antonietti, D. B. Kuang, B. Smarsly and Z. Yong, Angew. Chem., Int. Ed. 2004, 43, 4988-4992.

20 S.-g. Lee, Chem. Commun., 2006, 1049-1063.

21 Z. Ma, J. H. Yu and S. Dai, Adv. Mater., 2010, 22, 261-285.

22 S. Zhu, Y. Wu, Q. Chen, Z. Yu, C. Wang, S. Jin, Y. Ding and G. Wu, Green Chem., 2006, 8, 325-327.

23 D. Carriazo, M. C. Gutierrez, M. L. Ferrer and F. del Monte, Chem. Mater., 2010, 22, 6146-6152.

24 M. C. Gutierrez, D. Carriazo, C. O. Ania, J. B. Parra, M. L. Ferrer and F. del Monte, Energy Environ. Sci., 2011, 4, 3535-3544.

25 J. S. Lee, X. Q. Wang, H. M. Luo and S. Dai, Adv. Mater., 2010, 22, 1004.

26 J. P. Paraknowitsch, A. Thomas and M. Antonietti, J. Mater. Chem., 2010, 20, 6746-6758.

27 J. P. Paraknowitsch, J. Zhang, D. S. Su, A. Thomas and M. Antonietti, Adv. Mater., 2010, 22, 87.

28 J. P. Paraknowitsch, Y. Zhang and A. Thomas, J. Mater. Chem., 2011, 21, 15537.

29 J. S. Lee, X. Q. Wang, H. M. Luo, G. A. Baker and S. Dai, J. Am. Chem. Soc., 2009, 131, 4596.

30 L. Zhao, Y. S. Hu, H. Li, Z. X. Wang and L. Q. Chen, Adv. Mater., 2011, 23, 1385-1388.

31 T. Fukushima and T. Aida, Chem.-Eur. J., 2007, 13, 5048-5058.

32 P. Strasser, J. Comb. Chem., 2008, 10, 216-224.

33 K. Yaccato, R. Carhart, A. Hagemeyer, A. Lesik, P. Strasser, A. F. Volpe, H. Turner, H. Weinberg, R. K. Grasselli and C. Brooks, Appl. Catal., A, 2005, 296, 30-48.

34 G. Walter, R. Kranold, T. Gerber, J. Baldrian and M. Steinhart, J. Appl. Crystallogr., 1985, 18, 205-213.

$35 \mathrm{H}$. Schnablegger and Y. Singh, A Practical Guide to SAXS, Graz, 2006.

36 F. Jaouen, J. Herranz, M. Lefevre, J.-P. Dodelet, U. I. Kramm, I. Herrmann, P. Bogdanoff, J. Maruyama, T. Nagaoka, A. Garsuch, J. R. Dahn, T. Olson, S. Pylypenko, P. Atanassov and E. A. Ustinov, ACS Appl. Mater. Interfaces., 2009, 8, 1623-1639.

37 D. Villers, X. Acques-Bedard and J. P. Dodelet, J. Electrochem. Soc., 2004, 151, A1507-A1515.

38 G. Lalande, R. Cote, D. Guay, J. P. Dodelet, L. T. Weng and P. Bertrand, Electrochim. Acta, 1997, 42, 1379-1388.

39 G. Wu, Z. Chen, K. Artyushkova, F. H. Garzon and P. Zelenay, ECS Trans., 2008, 16, 159-170.

40 R. Bashyam and P. Zelenay, Nature, 2006, 443, 63-66. 